\title{
Vascularity of human atrioventricular valves: A myth or fact?
}

\author{
Narbada Saini, MSc, ${ }^{a}$ Uma Nahar Saikia, MD, ${ }^{\mathrm{b}}$ Daisy Sahni, PhD, ${ }^{\mathrm{a}}$ and Rana Sandip Singh, $\mathrm{MCh}^{\mathrm{c}}$
}

Background: Knowledge of heart valve vascularity is an important factor for understanding the valvular pathology and to develop tissue-engineered valves for repair procedures. Some investigators believe that blood vessels may exist in normal human heart valves whereas some recent publications have proposed that the presence of blood vessels in the valves is secondary to inflammation.

\begin{abstract}
Methods: Tissues from 60 normal formalin-fixed human hearts were examined microscopically for type, location, and number of vessels in atrioventricular valves. The age of the patient ranged from 10 to 70 years, and an attempt was made to study the age-related morphologic changes in atrioventricular valves.

Results: Of the 60 tricuspid and 60 mitral valves examined, 12 tricuspid (20\%) and 14 mitral $(23.33 \%)$ valves were found to have vessels without the presence of an inflammatory process. In tricuspid valves the vessels were observed mainly in the fibrosa layer with a range of 1 to 4 vessels, whereas in mitral valves the vessels were situated mainly in the spongiosa layer with a range of 1 to 2 vessels. The maximum vascularity was seen in the fourth decade of life, in which the vessels were found in $40 \%$ of both tricuspid and mitral valves. The mean transverse diameter of these vessels was $0.23 \pm 0.18 \mathrm{~mm}$, with a range of 0.06 to $0.79 \mathrm{~mm}$ in tricuspid valves, whereas it was $0.15 \pm 0.08 \mathrm{~mm}$, with a range of 0.04 to $0.4 \mathrm{~mm}$ in mitral valves. The capillaries (311 capillaries) were found scattered in the fibrosa and spongiosa with an average lumen area of $0.39 \pm 0.18 \mathrm{~mm}^{2}$.

Conclusions: The blood vessels in atrioventricular valves also can be seen in the absence of inflammation and are likely to be a necessary component of valve leaflets. Thus, when performing procedures involving in situ tissue engineering and valve repair the physician needs to be aware of the presence of these vessels in human heart valves. (J Thorac Cardiovasc Surg 2014;147:517-21)
\end{abstract}

Heart valves traditionally were thought to be avascular. ${ }^{1}$ Luschka ${ }^{2}$ was the first to show that heart valves contain a network of vessels in their broad portions, with small vessels running in their line of coaptation and a few vessels in their chordate tendinae. This led to a debate regarding the presence of vessels in mature, normal human heart valves. ${ }^{3}$ In many research reports, investigators have held to the belief that blood vessels may exist in normal human heart valves, ${ }^{4,5}$ whereas some publications have proposed that the presence of blood vessels in the valves is of pathologic origin. $^{6-8}$ It has been suggested that the valves normally are nonvascularized and that, if blood vessels are found, they are secondary to the inflammatory process in the leaflets ${ }^{6}$ or to a nonregression of normal fetal vascularization. ${ }^{4,5}$ Rheumatic heart disease is the most common cause of inflammation involving valve leaflets and shows

From the Department of Anatomy, ${ }^{\mathrm{a}}$ Department of Histopathology, ${ }^{\mathrm{b}}$ and Department of Cardiovascular and Thoracic Surgery, ${ }^{\mathrm{c}}$ Postgraduate Institute of Medical Education and Research, Chandigarh, India.

Supported by a grant from Indian Council of Medical Research, New Delhi, India. Disclosures: Authors have nothing to disclose with regard to commercial support. Received for publication Nov 29, 2012; revisions received March 14, 2013; accepted for publication March 20, 2013; available ahead of print April 22, 2013.

Address for reprints: Uma Nahar Saikia, MD, Department of Histopathology,

Postgraduate Institute of Medical Education and Research, Room 507, 5th Floor,

Chandigarh, India (E-mail: umasaikia@gmail.com).

$0022-5223 / \$ 36.00$

Copyright $\odot 2014$ by The American Association for Thoracic Surgery

http://dx.doi.org/10.1016/j.jtcvs.2013.03.031 neovascularization with or without inflammation. ${ }^{7,8}$ The vessels in rheumatic valve leaflets were found to be increased in density, thickness, and diameter compared with normal valve leaflets. ${ }^{7}$ Likewise, the occurrence of vessels in valves that show clinical or pathologic evidence of endocarditis can be attributed to inflammatory lesions. ${ }^{9}$

Previous studies have shown the presence of vessels in normal valve leaflets across multiple species, including human beings, with the help of perfusion patterns. ${ }^{6,10-13}$ There are scarce data regarding histomorphologic details of blood vessels in human valve leaflets. We analyzed 240 valve leaflets to characterize the presence of blood vessels in normal human tricuspid and mitral valves. In addition, we also studied age-related histomorphologic changes in atrioventicular valve apparatus of human hearts.

\section{MATERIALS AND METHODS}

The study material comprised 60 formalin-fixed human hearts (30 male hearts and 30 female hearts) obtained retrospectively from the Department of Histopathology at the Postgraduate Institute of Medical Education and Research (Chandigarh, India). There was no macroscopic developmental failure or pathologic change. All the hearts were taken from patients dying of noninflammatory conditions in the hospital and undergoing routine medical autopsies in the Department of Histopathology with duly informed consent from patient's relatives. The patients dying of inflammatory conditions including rheumatic heart disease, myocarditis, infective endocarditis, and autoimmune diseases involving the heart and patients with a history of coronary artery disease or ischemic heart disease were excluded from the study. The age range of the patients at time of death was 10 to 70 years, with an average age of $40.6 \pm 16.5$ years. 
In each heart, tricuspid and mitral valve apparatus were excised at anterior and posterior leaflets along with their chordate tendine and a part of the papillary muscle. These samples, including 120 tricuspid and 120 mitral valve apparatus, were fixed in $10 \%$ buffered formalin and processed routinely for paraffin embedding. In each case, $5-\mu \mathrm{m}$-thick sections were cut and stained with hematoxylin and eosin for histomorphology. A histochemical stain using Masson's trichrome was performed to determine the distribution of collagen and the extent of fibrosis, along with elastin Van Gieson stain for elastic and connective tissues and to characterize the vascularity of tissues. To assess the accumulation of mucopolysaccharide material, a periodic acid-Schiff-Alcian blue stain was performed on all the samples. Each section was examined for type, location, and number of vessels under a light microscope by 2 unbiased observers (U.N.S. and N.S.). The morphometry of the vessels was performed with the help of V-Test software (size 5.0; Video Test Company, St. Petersburg, Russia) and the selected sections were photographed using a Nikon charge-coupled device camera (Melville, NY). Qualitative data are expressed as numbers and percentages and quantitative data are expressed as ranges and means \pm standard deviation.

\section{RESULTS}

Light microscopic examination of the atrioventricular valve apparatus of human hearts showed that blood vessels in the form of veins, arterioles, small arteries, and capillaries were present in tricuspid and mitral valve apparatus as follows.

\section{Tricuspid Valve}

Of the 60 tricuspid valves examined, 12 valves $(20 \%)$ were found to have vessels with a male to female ratio of $4: 2$. The principal vessels in vascularized leaflets were veins and arterioles, followed by small arteries. The vessels appeared mainly in the fibrosa layer with a range of 1 to 4 vessels. The involvement of the spongiosa layer was less conspicuous. The mean transverse diameter of these vessels was $0.23 \pm 0.18 \mathrm{~mm}$, with a range of 0.06 to $0.79 \mathrm{~mm}$. No vessels were seen in the atrialis and ventricularis layers.

\section{Mitral Valve}

Of 60 mitral valves examined microscopically, blood vessels were found in $14(23.33 \%)$ samples with a male to female ratio of 3:4. The vessels were situated mainly in the spongiosa with a range of 1 to 2 vessels, and occasionally in the fibrosa (Figure 1). In 2 mitral valve leaflets, a single vessel was situated in the spongiosa layer and was projecting slightly toward the endothelial surface of the leaflet on the atrial side. None of the leaflets showed vessels in the ventricularis. The prominent vessels in these leaflets were veins and arterioles. The mean transverse diameter of the vessels was $0.15 \pm 0.08 \mathrm{~mm}$, with a range of 0.04 to $0.4 \mathrm{~mm}$. In 3 mitral valve leaflets, numerous capillaries (range, 3-11) were found scattered throughout the fibrosa and spongiosa (Figure 2). These capillaries were circular with a mean transverse diameter of $0.05 \pm 0.03 \mathrm{~mm}$ (range, 0.02-0.11 mm). There was no feature to suggest any inflammatory process in any of the valves. The capillaries appeared in groups with an average lumen area of $0.39 \pm 0.18 \mathrm{~mm}^{2}$.

The present study highlighted a relation between age and heart valve vascularity. The maximum vascularity was seen in the fourth decade of life, at which point vessels were found in $40 \%$ of both the tricuspid and mitral valves. Only $10 \%$ of mitral valves in the second decade showed vessels, whereas in the third decade no vessels were found in any of the atrioventricular valves. In the fifth decade, only $20 \%$ of the tricuspid and mitral valves showed vascularity. Vessels were found in $40 \%$ of the tricuspid and $30 \%$ of the mitral valves in the sixth decade, and in $20 \%$ of the tricuspid and $40 \%$ of the mitral valves in the seventh decade. In the seventh decade, blood vessels were found in one mitral chordae tendinae. In all the specimens examined, each papillary muscle was vascularized with at least 2 to 3 arterioles.

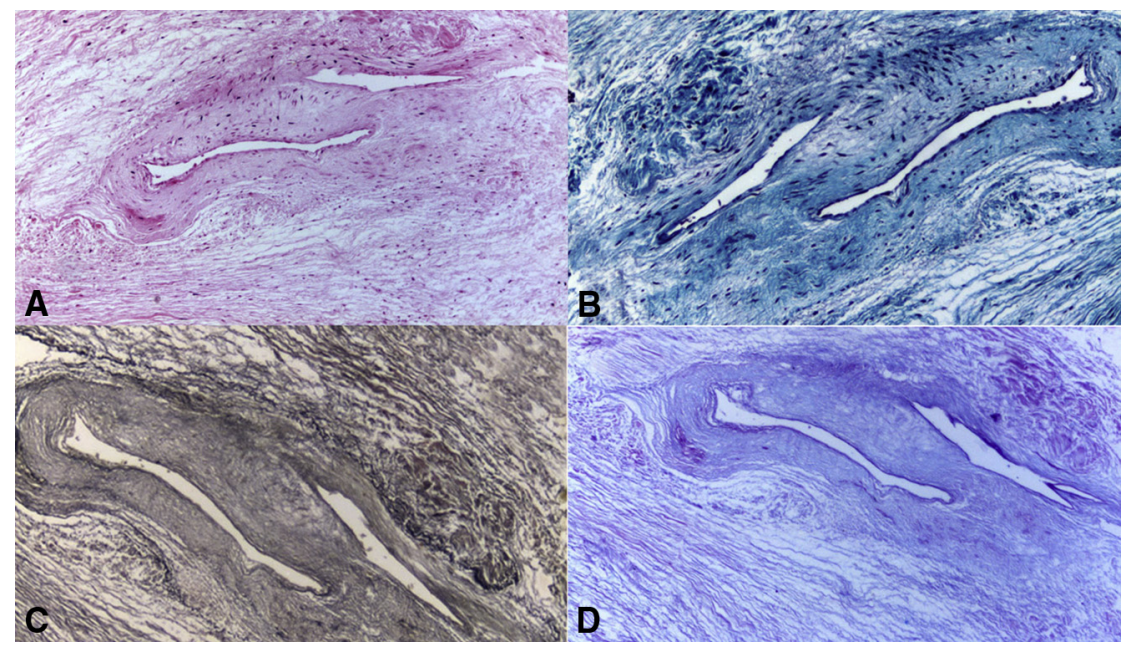

FIGURE 1. A, Microphotograph showing large-caliber veins in mitral valve leaflet (hematoxylin-eosin stain, $\times 10$ ). B, Masson's trichrome stain showing fibrosis with collagen deposition (magnification, $\times 10$ ). C, Elastin Van Gieson stain depicting internal elastic lamina of veins (magnification, $\times 10$ ). D, Alcian blue-periodic acid-Schiff stain showing degenerated mucopolysaccharide material accumulation (magnification, $\times 10$ ). 


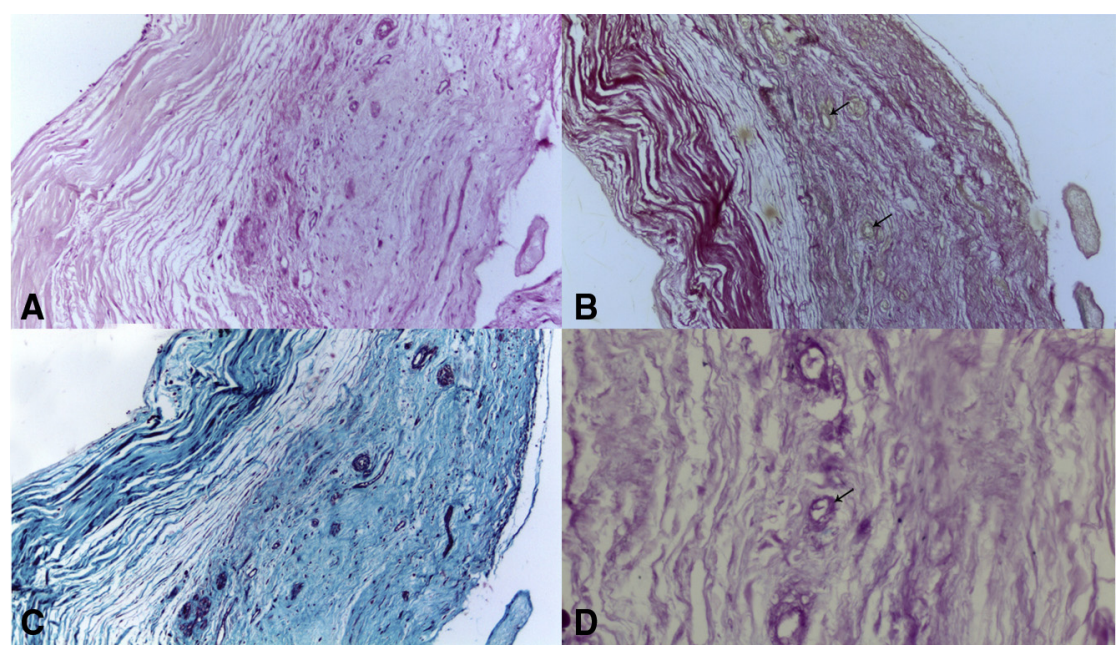

FIGURE 2. A, Microphotograph showing arterioles, capillaries, and venules in mitral valve leaflet (hematoxylin-eosin stain, $\times 10$ ). B, Elastin Van Gieson stain highlighting the arterioles (arrows) (magnification, $\times 10$ ). C, Masson's trichrome stain showing vessels of small caliber with mild fibrosis (magnification, $\times 10)$. D, Alcian blue-periodic acid-Schiff stain showing prominent arterioles with basement membrane and capillaries (arrow) (magnification, $\times 20)$.

In addition, we also observed moderate to marked mucopolysaccharide accumulation in these samples, which was more common in the mitral valves compared with the tricuspid valves. However, no specific distribution of blood vessels was seen in the tricuspid or mitral valves examined. Most of the vessels appeared to have a single endothelial layer and had an average lumen area of $0.50 \pm 0.54 \mathrm{~mm}^{2}$ in tricuspid valves and $0.39 \pm 0.55 \mathrm{~mm}^{2}$ in mitral valves.

\section{DISCUSSION}

Luschka $^{2}$ was the first to show that both atrioventricular and semilunar valves contain blood vessels, which subsequently was corroborated by many other investigators. ${ }^{3-6,14}$ For many years, heart valve vascularity has been the subject of investigation. One theory is that the blood vessels in human valves are present only when there is inflammation in the atrial or ventricular aspect of the leaflet ${ }^{6}$ or are the result of previous mild, and in most instances completely healed, inflammation. ${ }^{4}$ This also has been described that sometimes asymptomatic rheumatic fever or endocarditis that has completely healed becomes responsible for the formation of blood vessels in apparently normal atrioventricular valves. ${ }^{4,9}$ The stimuli for vessel formation in such instances are the chemotactic substances produced by inflammation, which stimulate the proliferation of endothelium of normal vessels. ${ }^{15}$ Another theory is that a small percentage of normal valves show a definite and well-organized system of blood vessels. ${ }^{6}$ The presence or absence of blood vessels in valves can become a basis of the pathogenesis of embolic valvular endocarditis. This is explained as follows, if the normal valves are vascular, then the bacterial emboli can enter the valve through these vessels and become arrested at the closing edge of the valve. ${ }^{6}$
We studied the vascularity of 60 anterior and 60 posterior leaflets of tricuspid and mitral valves along with their chordae tendinae and partial papillary muscles with no evident cardiac illness. Blood vessels in the form of veins, small arteries, arterioles, and capillaries were found in both valve leaflets in the absence of an inflammatory process. In $12 \%$ of tricuspid valve leaflets there were 1 to 4 vessels observed in the fibrosa layer, whereas in $23.33 \%$ of mitral valve leaflets there were 1 to 2 vessels present mainly in the spongiosa layer. There was no feature to suggest any inflammatory processes in these valves. A previous study performed using radiograph microscopy showed that $16 \%$ of the tricuspid and $10 \%$ of the mitral valve cusps were vascularized that were without any gross stigma of rheumatic disease. ${ }^{11} \mathrm{How}-$ ever, we found a higher percentage of vascularity in mitral valves compared with the tricuspid valves in our study. This possibly is because we performed a microscopic study, which increases the chances of finding blood vessels within the valves compared with other methods used by previous investigators such as a radiograph or perfusion study, which may not detect all types of vessels. In another histochemical study of normal mitral valve leaflets, ${ }^{16}$ blood vessels were found in $50 \%$ of valve leaflets and were distributed near the commissure areas of the auricularis and spongiosa, which was quite comparable with the distribution pattern of vessels in our study.

The transverse diameter of the predominant vessels in tricuspid valves was $0.23 \pm 0.18 \mathrm{~mm}$ (range, $0.06-0.79 \mathrm{~mm}$ ), whereas in mitral valves it was $0.15 \pm 0.08 \mathrm{~mm}$ (range, 0.04-0.4 mm). In a previous vascularity study of 50 mitral valves with no gross stigma of rheumatic disease, the wide range of transverse diameters measured was 0.05 to $0.5 \mathrm{~mm}$, and was comparable with our results. ${ }^{15}$ The vessels in the present study appeared to have a single endothelial 
layer with an average lumen area of $0.50 \pm 0.54 \mathrm{~mm}^{2}$ in tricuspid valves and $0.39 \pm 0.55 \mathrm{~mm}^{2}$ in mitral valves. Similarly, another pattern of vascularization study in 6 normal and 6 rheumatic mitral valves showed an average lumen area of $0.19 \pm 0.28 \mathrm{~mm}^{2}$ and $2.14 \pm 0.81 \mathrm{~mm}^{2}$, respectively, which was less than our results of normal mitral valves. ${ }^{7}$ This study supported the theory that vessels in rheumatic mitral valves are increased in density, thickness, and diameter compared with normal valve leaflets. ${ }^{7}$ Because we studied a wide range of cases in different age groups, our values were higher compared with previous results. However, because there is a paucity of histomorphologic studies of the presence of blood vessels in tricuspid leaflets because they are less likely to be involved in heart valves in various diseases other than rheumatic heart disease and endocarditis, a definite comparison was not possible.

We also studied the relation between age and heart valve vascularity. The maximum vascularity was seen in the fourth decade, followed by the fifth and sixth decades. The increased vascularity in the fourth decade possibly is related to the increased mucopolysaccharide accumulation. The vessels could be responsible for the infiltration of mucopolysaccharide within the valve leaflets. In addition, there was not much difference in the incidence of vessels in the tricuspid and mitral valve leaflets in each decade. In a previous study conducted by injecting dye into normal mitral valves in all age groups from birth to the ninth decade, a relationship was shown for the presence of vessels in the valves with age, but no accurate statistics were given in different age groups, which made us unable to compare our results. ${ }^{4}$ The blood vessels were found in one of the mitral chordae tendinae in a 65-year-old man. The presence of vessels characterize the chordae tendineae as complex components that work in coordination with the papillary muscles and valve leaflets to ensure proper physiological valve functions. This finding is in accordance with a previous study on structural characterization of chordae tendinae in native porcine mitral valves that also showed that blood vessels were present in the longitudinal and circumferential directions of the chordae. ${ }^{17}$ The histologic sections in our study showed that at least 2 to 3 arterioles were present in each papillary muscle, which was supported by another study conducted on 54 human hearts using coronary angiography, arterial casts, and histologic sections. ${ }^{11}$

The present study also examined age-related degenerative changes in the form of disruption of collagen bundles within fibrosa and accumulation of mucopolysaccharide in spongiosa and fibrosa layers of valve leaflets. The majority of the vessels were reported in those sections, which showed moderate to marked grades of accumulation of mucopolysaccharide. The question is whether the vessels that are present in valves are responsible for mucopolysaccharide material and bacterial emboli infiltration within the leaflets, which leads to valvular insufficiency and endocarditis, respectively. The increase in the extent of mucopolysaccharide material along with thickened fibrosa usually accompanies floppy or myxomatous valve syndrome. ${ }^{18}$ This suggests that the presence of vessels does not depend solely on the inflammation of valves but also depends on degenerative changes, including myxomatous degeneration within the valve leaflets.

We determined the relationship between vascularity and degenerative changes in aging heart valves. In addition, in most cases previous studies did not include the histomorphologic aspect of vessels in valve leaflets. In all the microscopically examined sections of tricuspid and mitral valve leaflets, the basic structural change was collagen degeneration along with accumulation of mucopolysaccharide and hyalinization of chordae tendinae. Inflammation, calcification, Aschoff nodules, and endocardial reduplication were not found in any of the tricuspid and mitral valve leaflets.

Thus, we conclude that the vascularity of artioventricular valve leaflets can be seen in the absence of inflammation and has a relationship with age-related degenerative changes of heart valves. The blood vessels in atrioventricular valves are not vestigial holdovers, but likely are a necessary component that requires the development of tissue-engineered valves that resemble a native valve. The procedures involving tissue engineering are promising for autologous and functional heart valve replacements. It is important to consider the presence of vessels in atrioventricular valves during their repair and remodeling to assess long-term outcomes. Despite successful tissue engineering, the implanted tissues are incapable of stimulating vasculature for their survival. Thus, if vascularity in atrioventricular valve leaflets is considered, it may be easy to develop an engineered valve resembling a native valve that may have the potential to last longer. This information is useful not only from a histologic point of view but also for its bearing on the interpretation of disease processes in cardiovascular interventions and surgeries.

The present study was limited because of the small number of cases and because surgical tissue was not included. The valve vascularity was not studied by immunohistochemistry and we did not compare the valve leaflet vascularity in normal and diseased valve leaflets, where the vascularity is reported to be increased secondary to inflammation.

We acknowledge the excellent technical assistance of Mr Charan Singh, Department of Histopathology, and Mr Pardeep Singh, Department of Anatomy, Postgraduate Institute of Medical Education and Research, Chandigarh, India.

\section{References}

1. Millington SC, Meir A, Lawrence L, Stolinski C. Structure of chordae tendineae in the left ventricle of the human heart. J Anat. 1998;192:573-81.

2. Luschka H. Das Endocardium und die Endocarditis. Virch Arch. 1852;4:171-92. 3. Bayne JS. Blood vessels of the heart valves. Am J Anat. 1917;21:449. 
4. Gross L. Significance of blood vessels in human heart valves. Am Heart J. 1937; 13:275-96.

5. Ritter SA, Gross L, Kugel MA. Blood vessels in the valves of normal human hearts: from a study of 700 human hearts. Am Heart J. 1928;3:433-46.

6. Dow DR, Harper WF. The vascularity of the valves of the human heart. J Anat. 1932;66:610-7.

7. Salhiyyah K, Kotit P, Sarathchandra P, Chester AH, Yacoub M. Rheumatic valve disease is associated with neovascularisation of mitral valve leaflets. Poster presented at: The Society of Heart Valve Disease, 6th Biennial Meeting; June 25-28, 2011; Barcelona, Spain.

8. Sampaio RO, Fae KC, Demarchi LMF, Pomerantzeff PMA, Aiello VD, Spina GS, et al. Rheumatic heart disease: 15 years of clinical and immunological follow-up. Vasc Health Risk Manag. 2007;3:1007-17.

9. Harper WF. The blood supply of heart valves in relation to endocarditis. J Anat. 1938;73:94-111.

10. Swanson JC, Davis LR, Arata K, Briones EP, Bothe W, Itoh A, et al. Characterization of mitral valve anterior leaflet perfusion patterns. J Heart Valve Dis. 2009; 18:488-95.
11. Clarke JA. An X-ray microscopic study of blood supply to the valves of the human heart. Br Heart J. 1965;27:420-3.

12. Wearn JT, Bromer AW, Zschiesche LJ. The incidence of blood vessels of heart valves. Am J Heart. 1936;11:22-33.

13. Duran CM, Gunning AJ. The vascularization of the heart valves: a comparative study. Cardiovasc Res. 1968;2:290-6.

14. Wearn JT, Zschiesche LJ. The extent of capillary bed of heart. J Exp Med. 1928; 47:273-90.

15. Koletsky S. Gross vascularity of the mitral valve as a stigma of rheumatic heart disease. Am J Pathol. 1946;22:351-67.

16. I-Ida T, Tamura K, Tanaka S, Asano G. [Blood vessels in normal and abnormal mitral valve leaflets]. J Nippon Med Sch. 2001;68:171-80.

17. Ritchie J, Warnock JN, Yoganathan AP. Structural characterization of the chordae tendineae in native porcine mitral valves. Ann Thorac Surg. 2005;80: 189-97.

18. McCarthy KP, Ring L, Rana BS. Anatomy of the mitral valve: understanding the mitral valve complex in mitral regurgitation. Eur J Echocardiogr. 2010; 11:13-9. 